\title{
Effects of Hall current and heat absorption on MHD flow with variable wall temperature and mass diffusion.
}

\author{
U.S. Rajput ${ }^{{ }^{*}}$, Neetu Kanaujia ${ }^{2}$ \\ ${ }^{1}$ Department of Mathematics and Astronomy, University of Lucknow, Lucknow, India \\ ${ }^{2}$ Department of Mathematics and Astronomy, University of Lucknow, Lucknow, India \\ *Corresponding Author: rajputneetulko@gmail.com
}

Available online at: www.isroset.org

Received: 03/Aug/2018, Accepted 23/Aug/2018, Online 31/Oct/2018

\begin{abstract}
The present paper analyses the effects of Hall current and heat absorption on flow model. The flow is along an exponentially accelerated vertical plate with variable heat transfer and mass diffusion. The fluid considered is viscous, incompressible and electrically conducting. The magnetic field is applied perpendicular to the plate. The problems related to rotation, Hall current, heat transfer and mass diffusion are being studied due to their applications in many processes. This is the motivation for the study of this model. Velocity profiles, temperature profiles and concentration profiles are obtained by Laplace transform technique for different parameters like rotation parameter, magnetic field parameter, heat absorption parameter, Hall parameter, acceleration parameter, Prandtl number, the angle of inclination of plate, chemical reaction parameter, acceleration parameter and time. The numerical data obtained are discussed with the help of graphs and tables. The numerical values obtained for skin-friction and Nusselt number have been tabulated.
\end{abstract}

Keywords - MHD flow, Heat absorption fluid, Variable wall temperature, Mass diffusion, Hall current

\section{INTRODUCTION}

Magnetohydrodynamic flow with Hall current is studied in different areas such as geophysics, the oceanography, and industrial engineering. Many researchers have studied different types of models, of which some are mentioned here. Chemical reaction in the peristaltic motion of MHD couple stress fluid in the channel with Soret and Dufour effects was investigated by Tasawar Hayat et al. [1]. Aboelahad and Elbarbary [2] have analyzed Hall current effect on magneto hydrodynamic free convection flow past a semi- infinite vertical plate with mass transfer. Seddeek [3] has studied the effects of chemical reaction, thermophoresis and variable viscosity on steady hydro magnetic flow with heat and mass transfer. MHD mixed convection interaction with thermal radiation was explored by Makinde [4]. Shehzad et al. [5] have investigated three - dimensional MHD flow of Casson fluid in porous medium with heat generation.

Zueco and Ahmed [6] examined the combined heat and mass transfer by mixed convection MHD flow along a porous plate with chemical reaction in presence of heat source. Effects of viscous dissipation and heat source on unsteady MHD flow over a stretching sheet was considered by Reddy et al. [7]. Seth et al. [8] have discussed Hall effects on unsteady MHD natural convection flow of a heat absorbing fluid past an accelerated moving vertical plate with ramped temperature. Samad et al. [9] have worked on MHD free convection and mass transfer flow through a vertical oscillatory porous plate with Hall, ion-slip currents and heat source in a rotating system. Hayat, et al. [10] have investigated Hall effect on unsteady flow due to noncoaxicially rotating disk and a fluid at infinity. Manivannan et al. [11] have described radiation and chemical reaction effects on isothermal vertical oscillating plate with variable mass diffusion.

Earlier we [12] worked on MHD flow past a vertical plate with variable temperature and mass diffusion in the presence of Hall current. Dahake and Dubewar [13] have investigated effects of radiation on magnetohydrodynamic convection flow past an impulsively started vertical plate submersed in a porous medium with suction. The purpose of the present investigation is to study effects of Hall current and heat absorption on MHD flow with variable wall temperature and mass diffusion. The model has been solved using the Laplace transforms technique. The results are shown with the help of graphs and table.

\section{Mathematical ANALYSIS}

The $\mathrm{x}$ axis is taken in upward direction and y normal to it. A transverse magnetic field of strength $B_{0}$ is applied on the plate which is inclined at an angle $\alpha$ from the vertical. The magnetic Reynolds number is considered to be small so that the induced magnetic field is neglected. Initially, it has been considered that the plate and the fluid are at the same 
temperature $\left(T_{\infty}\right)$. The species concentration in the fluid is taken as $C_{\infty}$. At time $\mathrm{t}>0$, the plate is accelerated exponentially taking velocity $u=u_{0} \exp (b t)$ in its own plane. Also the temperature and the concentration near the plate are raised linearly with respect to time. The governing equations are as under:

$\frac{\partial u}{\partial t}-2 \Omega w=v \frac{\partial^{2} u}{\partial y^{2}}+g \beta \operatorname{Cos} \alpha\left(T-T_{\infty}\right)$

$+g \beta^{*} \operatorname{Cos} \alpha\left(C-C_{\infty}\right)-\frac{\sigma B_{0}{ }^{2}}{\rho\left(1+m^{2}\right)}(u+m w)$,

$\frac{\partial w}{\partial t}+2 \Omega u=v \frac{\partial^{2} w}{\partial y^{2}}-\frac{\sigma B_{0}{ }^{2}}{\rho\left(1+m^{2}\right)}(w-m u)$,

$\frac{\partial C}{\partial t}=D \frac{\partial^{2} C}{\partial y^{2}}$,

$\rho C_{p} \frac{\partial T}{\partial t}=k \frac{\partial^{2} T}{\partial y^{2}}-Q\left(T-T_{\infty}\right)$.

The initial and boundary conditions are

$t \leq 0: u=0, w=0, T=T_{\infty}, C=C_{\infty}$ for every $y$,

$t>0: u=u_{0} e^{b t}, w=0, T=T+\left(T_{w}-T_{\infty}\right) \frac{u_{0}{ }^{2} t}{v}, C=C_{\infty}+\left(C_{w}-C_{\infty}\right) \frac{u_{0}{ }^{2} t}{v}$ at $\left.y=0.\right\}$

$u \rightarrow 0, w \rightarrow 0, T \rightarrow T_{\infty}, C \rightarrow C_{\infty}$ as $y \rightarrow \infty$

Here $u$ is the primary velocity, $w$-the secondary velocity, $g$ the acceleration due to gravity, $\beta$-volumetric coefficient of thermal expansion, $b$ - acceleration parameter, $t$ - time, $m$ is the Hall parameter with $\omega_{e}$ - cyclotron frequency of electrons and $\tau_{e}$-electron collision time, $K$-the permeability parameter, $T$ - temperature of the fluid, $\beta^{*}$ - volumetric coefficient of concentration expansion, $C$ - species concentration in the fluid, $v$-the kinematic viscosity, $\rho$-the density, $C_{P}$ - the specific heat at constant pressure, $k$ - thermal conductivity of the fluid, $D$ - the mass diffusion coefficient, $T_{w}$-temperature of the plate at $y=0, C_{w}$-species concentration at the plate $y$ $=0, B_{0}$ - the uniform magnetic field, $K_{c}$-chemical reaction, $\sigma$ - electrical conductivity.

$\bar{y}=\frac{y u_{0}}{v}, \bar{u}=\frac{u}{u_{0}}, \bar{w}=\frac{w}{u_{0}}, \theta=\frac{\left(T-T_{\infty}\right)}{\left(T_{w}-T_{\infty}\right)}, S c=\frac{v}{D}, \mu=\rho v$,

$\operatorname{Pr}=\frac{\mu C_{p}}{k}, H=\frac{Q v}{u_{0}^{2} \rho C_{p}}, \bar{\Omega}=\frac{v \Omega}{u_{0}^{2}} G r=\frac{g \beta v\left(T_{w}-T_{\infty}\right)}{u_{0}{ }^{3}}$,

$M=\frac{\sigma B_{0}^{2} v}{\rho u_{0}^{2}}, G m=\frac{g \beta^{*} v\left(C_{w}-C_{\infty}\right)}{u_{0}{ }^{3}}, \bar{C}=\frac{\left(C-C_{\infty}\right)}{\left(C_{w}-C_{\infty}\right)}, \bar{t}=\frac{t u_{0}^{2}}{v}$.

where $\bar{u}$ - the dimensionless primary velocity, $\bar{w}$ - the secondary velocity, $\bar{b}$ - dimensionless acceleration parameter, $t$ - dimensionless time, $\theta$ - the dimensionless temperature, $\bar{C}$ - the dimensionless concentration, $\bar{K}$ - the dimensionless permeability parameter, $\Omega$ - rotation parameter, $\mathrm{H}$ - heat absorption parameter, $G r$ - thermal Grashof number, $G m$ - mass Grashof number, $K_{c}$ - Chemical reaction parameter, $\mu$ - the coefficient of viscosity, $\mathrm{Pr}$ - the Prandtl number, $S c$ - the Schmidt number, $M$ - the magnetic parameter.

The following non-dimensional quantities are introduced to transform equations (1), (2), (3) and (4) into dimensionless form:

The dimension less flow model becomes:

$\frac{\partial \bar{u}}{\partial \bar{t}}-2 \bar{\Omega} \bar{w}=\frac{\partial^{2} \bar{u}}{\partial \bar{y}^{2}}+G r \operatorname{Cos} \alpha \theta+G m \operatorname{Cos} \beta \bar{C}-\frac{M(\bar{u}+m \bar{w})}{\left(1+m^{2}\right)}$

$\frac{\partial \bar{w}}{\partial \bar{t}}+2 \bar{\Omega} \bar{u}=\frac{\partial^{2} \bar{w}}{\partial \bar{y}^{2}}-\frac{M(\bar{w}-m \bar{u})}{\left(1+m^{2}\right)}$,

$\frac{\partial \bar{C}}{\partial \bar{t}}=\frac{1}{S c} \frac{\partial^{2} \bar{C}}{\partial \bar{y}^{2}}$,

$\frac{\partial \theta}{\partial \bar{t}}=\frac{1}{\operatorname{Pr}} \frac{\partial^{2} \theta}{\partial \bar{y}^{2}}-H \theta$

The corresponding boundary conditions become

$\bar{t} \leq 0: \bar{u}=0, \bar{w}=0, \quad \theta=0, \bar{C}=0$ for every $\bar{y}$.

$\bar{t}>0: \bar{u}=e^{b t}, \bar{w}=0, \theta=\bar{t}, \bar{C}=\bar{t}$ at $\bar{y}=0$,

$\bar{u} \rightarrow 0, \bar{w} \rightarrow 0, \theta \rightarrow 0, \bar{C} \rightarrow 0$ as $\bar{y} \rightarrow \infty$.

Dropping bars in the above equations and combined equation (7) and (8) by using $(q=u+i w)$, the model becomes

$\frac{\partial q}{\partial t}=\frac{\partial^{2} q}{\partial y^{2}}+G r \operatorname{Cos} \alpha \theta+G m \operatorname{Cos} \alpha C$

$-\left(\frac{M}{1+m^{2}}(1-m i)+2 i \Omega\right) q$,

$\frac{\partial C}{\partial t}=\frac{1}{S c} \frac{\partial^{2} C}{\partial y^{2}}$,

$\frac{\partial \theta}{\partial t}=\frac{1}{\operatorname{Pr}} \frac{\partial^{2} \theta}{\partial y^{2}}-H \theta$

Finally, the boundary conditions become:

$\left.\begin{array}{l}t \leq 0: q=0, \theta=0, C=0, \text { for every } y \\ t>0: q=e^{b t}, \theta=t, C=t \text { at } y=0 \\ q \rightarrow 0, \theta \rightarrow 0, C \rightarrow 0, \text { as } y \rightarrow \infty .\end{array}\right\}$

The dimensionless governing equations (12) to (14), subject to the boundary conditions (15), are solved by the usual 
Laplace - transform technique. The solution obtained is as under:

$$
\begin{aligned}
& q=\frac{1}{2} \exp (b t-\sqrt{a+b} y) A_{33}+\frac{1}{4}\left\{\frac { G r \operatorname { C o s } \alpha } { ( a + H \operatorname { P r } ) ^ { 2 } } \left[( a t + \operatorname { P r } ( 1 + H t ) - 1 ) \left(e^{-\sqrt{a} y} A_{3}\right.\right.\right. \\
& \left.-\exp (-\sqrt{H \operatorname{Pr}} y) A_{11}\right)+\exp (-\sqrt{a} y) A_{4} \sqrt{a}\left(1+\frac{H \operatorname{Pr}}{a}\right)+A_{14}(1-\operatorname{Pr})\left(A_{5}-A_{12}\right) \\
& \left.-\frac{1}{2 i \sqrt{H}} y \exp (-\sqrt{H \operatorname{Pr}} y i) A_{10} \sqrt{\operatorname{Pr}}(H \operatorname{Pr}+a)\right]+\frac{G m \operatorname{Cos} \alpha}{a^{2}}\left[2 A_{6} \exp (-\sqrt{a} y)(1-a t)\right. \\
& \left.+\exp (-\sqrt{a} y)\left(y \sqrt{a} A_{8}+2 A_{9} S c\right)+2 A_{15} A_{7}(1-S c)\right]-\frac{2 G m \operatorname{Cos} \alpha}{a^{2} \sqrt{\pi}}\left[2 a y \sqrt{t S c} \exp \left(-\frac{y^{2} S c}{4 t}\right)\right. \\
& \left.\left.+A_{16} \sqrt{\pi}\left(a y^{2} S c+2 a t+2 S c-2\right)+A_{13} A_{15} \sqrt{\pi}(1-S c)\right]\right\} .
\end{aligned}
$$$$
C=t\left\{\left(1+\frac{y^{2} S c}{2 t}\right) \operatorname{erfc}\left[\frac{\sqrt{S c}}{2 \sqrt{t}}\right]-\frac{y \sqrt{S c}}{\sqrt{\pi \sqrt{t}}} e^{-\frac{y^{2}}{4 t}} S c\right\},
$$$$
\theta=\frac{1}{4 H i} \exp (-y i \sqrt{H P r})\left\{\begin{array}{l}
-2 i \sqrt{H} t\left(A_{1}-\exp (-y i \sqrt{H P r}) A_{2}-2\right) \\
+y \sqrt{\operatorname{Pr}}\left(A_{1}+\exp (-y i \sqrt{H P r}) A_{2}-2\right)
\end{array}\right\} .
$$

\section{1 SKIN FRACTION}

The dimensionless skin friction at the plate $y=0$ is computed

$$
\text { by } \quad\left(\frac{d q}{d y}\right)_{y=0}=\tau_{x}+i \tau_{z}
$$

\section{2 NUSSELT NUMBER}

$$
\begin{gathered}
\quad N u=\left(\frac{\partial \theta}{\partial y}\right)_{y=0} \\
=\frac{1}{4 \sqrt{-H}} \exp \left(-B_{3} y\right)\left[-B_{3}\left(2 \sqrt{-H} t+\sqrt{\operatorname{Pr}} y-\left(2 \sqrt{t} B_{1}\right) \operatorname{ErfB} B_{1}+\exp \left(2 B_{3} y\right)\left\{\left(2 \sqrt{t B_{2}}\right) \operatorname{ErfB} B_{2}\right.\right.\right. \\
-2 \sqrt{-H} t-\sqrt{\operatorname{Pr} y} y)+\left(-\sqrt{\operatorname{Pr}}+\exp \left(2 B_{3} y\right)\left[\sqrt{\operatorname{Pr}}+4 \sqrt{-H} B_{3} t+2 \sqrt{\operatorname{Pr}} B_{3} y-\sqrt{\operatorname{Pr}} \operatorname{ErfB} B_{2}\right]\right. \\
-\frac{\sqrt{\operatorname{Pr}}}{\sqrt{\pi}}\left[\exp \left(-B_{1}{ }^{2}\right) 2 B_{1}+\exp \left(2 B_{3} y-B_{2}{ }^{2}\right)\left(2 B_{3}+\sqrt{\operatorname{Pr}} y\right)-\sqrt{\operatorname{Pr}} \operatorname{Erf}\left[B_{1}\right]\right. \\
\left.\left.-2 \exp \left(2 B_{3} y\right) B_{3}\left(2 \sqrt{t} B_{2}\right) \operatorname{Erf}\left[B_{2}\right]\right)\right] .
\end{gathered}
$$

\section{INTERPRETATION OF RESULTS}

The velocity and skin friction have been computed for various parameter like, rotation parameter $(\Omega)$, magnetic field parameter $(M)$, heat absorption parameter $(H)$, Hall parameter $(m)$, Prandtl number $(P r)$, the angle of inclination of plate $(\propto)$, chemical reaction parameter $\left(K_{0}\right)$, acceleration parameter $(b)$ and time $(t)$. Figures 1,2 , and 4 show that primary velocity decreases when $\propto, H$, and $\Omega$ are increased. Also figure 3 shows that primary velocity increases when $b$ is increased. Further, it is analyzed from figure 5 and 6 that secondary velocity decreases when $\propto$ and $H$ are increased. Figure 7 and 8 show that secondary velocity increases when $b$ and $\Omega$ are increased. From table -1 it is deduced that $\tau_{x}$ increases with increase in $\Omega$ and it decreases when $\alpha, H$ and $b$ are increased. The value of $\tau_{z}$ increases with increase in $\Omega$, and $M$. Further, it decreases when $\alpha$ and $b$ are increased. It has been observed that the Nusselt number decreases with increase in Prandtl number and time. But it increases with increasing heat generation parameter.

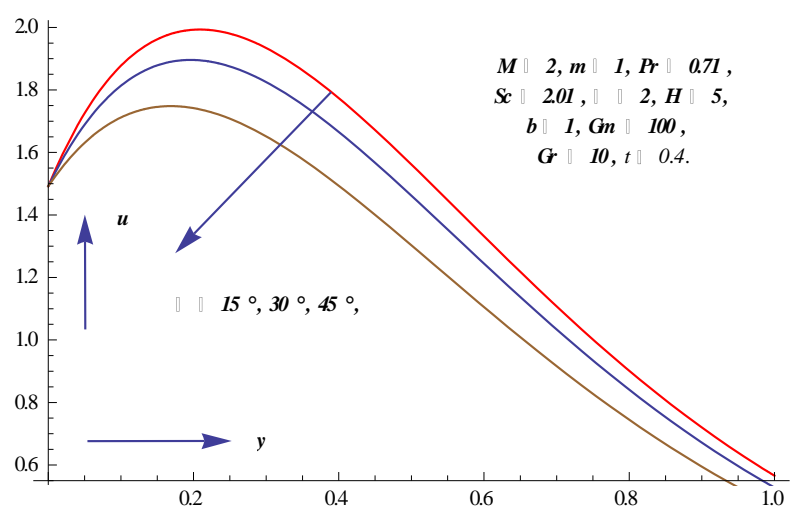

Figure 1. $u$ vs $y$ as for different values of $\propto$.

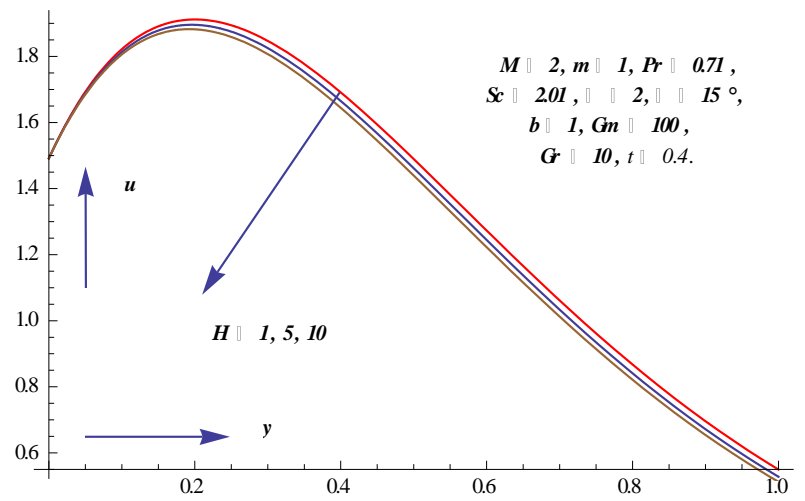

Figure 2. $u$ vs $y$ as for different values of $\mathrm{H}$.

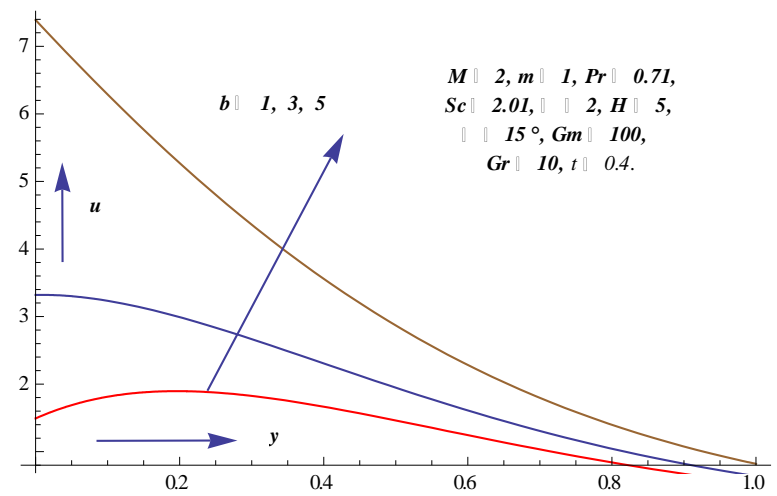

Figure 3. $u$ vs $y$ as for different values of $\mathrm{b}$. 


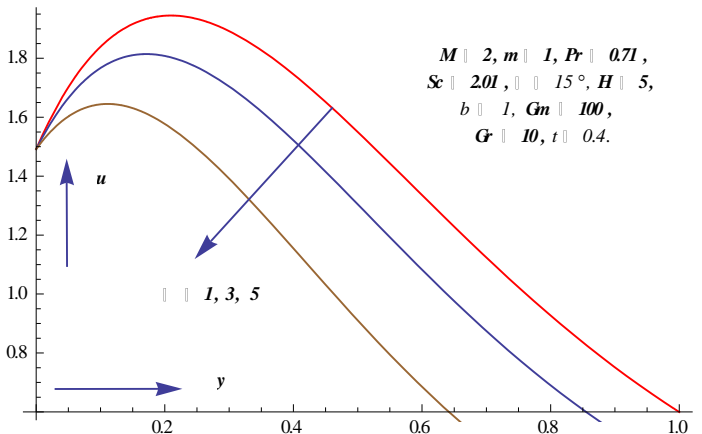

Figure 4. $u$ vs $y$ as for different values of $\Omega$.

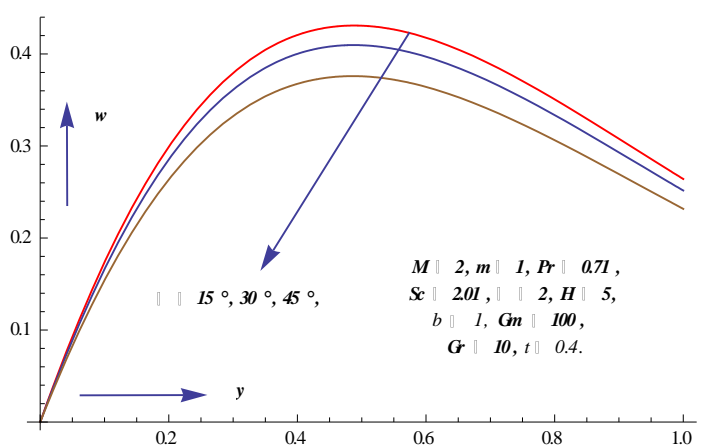

Figure 5. $u$ vs $y$ as for different values of $\propto$.

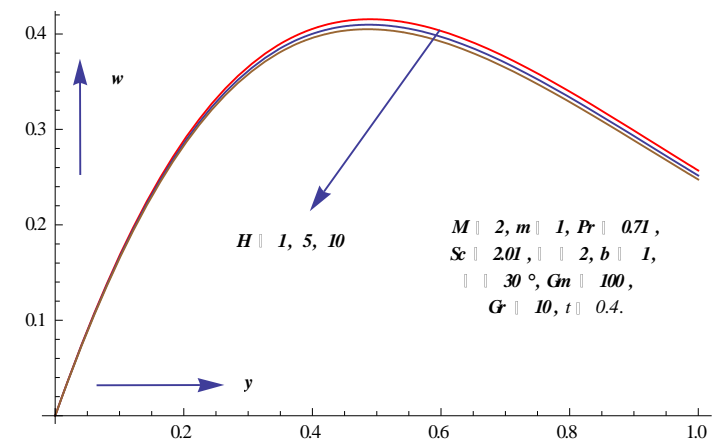

Figure 6. $u$ vs $y$ as for different values of $\mathrm{H}$.

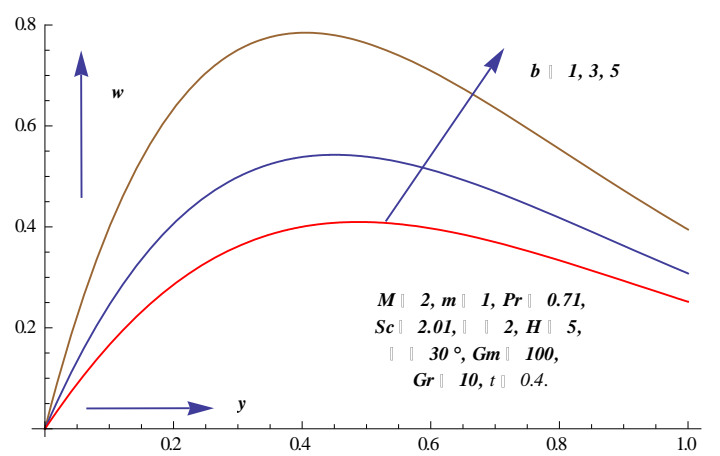

Figure 7. $u$ vs $y$ as for different values of $b$.

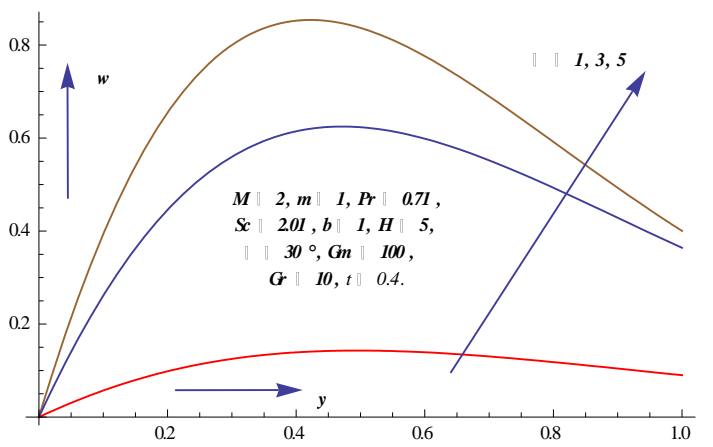

Figure 8. $u v s y$ as for different values of $\Omega$.

\section{CONCLUSION}

It is observed that the primary velocity decreases with increasing the values of angle of inclination of plate, heat absorption, and rotation parameter. However it increases with acceleration parameter. Further, it is analyzed that secondary velocity decreases when angle of inclination of plate and heat absorption are increased. Secondary velocity increases when acceleration parameter and rotation parameter are increased.

Table - 1 Skin fraction

\begin{tabular}{|l|l|l|l|l|l|l|l|l|l|l|l|l|}
\hline $\boldsymbol{m}$ & $\boldsymbol{G r}$ & $\boldsymbol{G} \boldsymbol{M}$ & $\boldsymbol{M}$ (degree) & $\boldsymbol{H}$ & $\boldsymbol{S c}$ & $\boldsymbol{P r}$ & $\Omega$ & $B$ & $\boldsymbol{t}$ & $\tau_{x}$ & $\tau_{z}$ \\
\hline 1.0 & 10 & 100 & 2.0 & $\mathbf{1 5}$ & -5 & 2.01 & 0.71 & 2.0 & 1 & 0.4 & $\mathbf{5 . 2 1 6 8}$ & $\mathbf{- 1 . 9 2 4 9}$ \\
\hline 1.0 & 10 & 100 & 2.0 & $\mathbf{3 0}$ & -5 & 2.01 & 0.71 & 2.0 & 1 & 0.4 & $\mathbf{4 . 4 2 5 2}$ & $\mathbf{- 1 . 8 4 6 8}$ \\
\hline 1.0 & 10 & 100 & 2.0 & $\mathbf{4 5}$ & -5 & 2.01 & 0.71 & 2.0 & 1 & 0.4 & $\mathbf{3 . 1 6 5 9}$ & $\mathbf{- 1 . 7 2 2 6}$ \\
\hline 1.0 & 10 & 100 & 2.0 & 30 & $\mathbf{- 1}$ & 2.01 & 0.71 & 2.0 & 1 & 0.4 & $\mathbf{4 . 5 1 4 1}$ & $\mathbf{- 1 . 8 6 2 6}$ \\
\hline 1.0 & 10 & 100 & 2.0 & 30 & $\mathbf{- 5}$ & 2.01 & 0.71 & 2.0 & 1 & 0.4 & $\mathbf{4 . 4 2 5 2}$ & $\mathbf{- 1 . 8 4 6 8}$ \\
\hline 1.0 & 10 & 100 & 2.0 & 30 & $\mathbf{- 1 0}$ & 2.01 & 0.71 & 2.0 & 1 & 0.4 & $\mathbf{4 . 3 4 8 9}$ & $\mathbf{- 1 . 8 3 3 4}$ \\
\hline 1.0 & 10 & 100 & 2.0 & 1.0 & -5 & 2.01 & 0.71 & $\mathbf{1 . 0}$ & 1 & 0.4 & $\mathbf{4 . 6 8 6 9}$ & $\mathbf{- 0 . 6 3 2 7}$ \\
\hline 1.0 & 10 & 100 & 2.0 & 1.0 & -5 & 2.01 & 0.71 & $\mathbf{3 . 0}$ & 1 & 0.4 & $\mathbf{3 . 9 4 9 9}$ & $\mathbf{- 2 . 9 2 0 1}$ \\
\hline 1.0 & 10 & 100 & 2.0 & 1.0 & -5 & 2.01 & 0.71 & $\mathbf{5 . 0}$ & 1 & 0.4 & $\mathbf{2 . 6 8 8 1}$ & $\mathbf{- 4 . 4 8 0 4}$ \\
\hline 1.0 & 10 & 100 & 2.0 & 1.0 & 1.0 & 2.01 & 0.71 & 2.0 & $\mathbf{3}$ & 0.4 & $\mathbf{0 . 0 4 5 0}$ & $\mathbf{- 2 . 8 2 9 5}$ \\
\hline 1.0 & 10 & 100 & 2.0 & 1.0 & 1.0 & 2.01 & 0.71 & 2.0 & $\mathbf{5}$ & 0.4 & $\mathbf{- 1 1 . 3 2 7}$ & $\mathbf{- 4 . 7 7 8 5}$ \\
\hline
\end{tabular}


Table - 2 Nusselt number

\begin{tabular}{|c|c|c|c|}
\hline $\boldsymbol{P r}$ & $\boldsymbol{H}$ & $\boldsymbol{t}$ & $\boldsymbol{N u}$ \\
\hline $\mathbf{7 . 0}$ & 1.0 & 0.1 & -0.9122 \\
\hline $\mathbf{1 3 . 0}$ & 1.0 & 0.1 & -1.2432 \\
\hline 0.71 & $\mathbf{5 . 0}$ & 0.1 & -0.2478 \\
\hline 0.71 & $\mathbf{1 0}$ & 0.1 & -0.1887 \\
\hline 0.71 & 1.0 & $\mathbf{0 . 2}$ & -0.3962 \\
\hline 0.71 & 1.0 & $\mathbf{0 . 3}$ & -0.4670 \\
\hline
\end{tabular}

\section{REFERENCES}

[1] Tasawar Hayata, Sabia Asghara, Anum Tanveera, Ahmed Alsaedib, "Chemical reaction in peristaltic motion of MHD couple stress fluid in channel with Soret and Dufour effects', Results in Physics, 10, 69 - 80 (Elsevier), 2018.

[2] Emad M. Aboelahad, Elsayel, M. E. Elbarbary, "Hall current effect on magneto hydrodynamic free convection flow past a semiinfinite vertical plate with mass transfer", international journal of engineering science, 1641-1652, 2001.

[3] M. A. Seddek, "Finite-element method for the effects of chemical reaction, variable viscosity, thermophoresis and heat generation/absorption on a boundary-layer hydro magnetic flow with heat and mass transfer over a heat surface". Acta Mech, 177, pp. 1-18, 2005 .

[4] O.D. Makinde, MHD mixed- convection interaction with thermal radiation and $n^{\text {th }}$ order chemical reaction past a vertical porous plate embedded in a porous medium, chemical engineering communications, 2010.

[5] S. A Shehzad, T. Hayat and A. Alsaedi, "Three-dimensional MHD flow of Casson fluid in porous medium with heat generation", Journal of Applied Fluid Mechanics, Vol. 9, No. 1, pp. 215-223, 2016.

[6] J. Zueco, and S. Ahmed, "Combined heat and mass transfer by mixed convection MHD flow along a porous plate with chemical reaction in presence of heat source", Applied. Math. Mech". 31 (10), 1-14, 2010.

[7] M G Reddy, P Padma and B Shankar, "Effects of viscous dissipation and heat source on unsteady MHD flow over a stretching sheet", Ain Shams Engineering Journal, 6, 1195-1201, 2015.

[8] Seth G. S., Sharma R. and Hussain S. M., "Hall effects on unsteady MHD natural convection flow of a heat absorbing fluid past an accelerated moving vertical plate with ramped temperature", Emirates Journal for Engineering Research, 19 (2), pp.19-32, 2014.

[9] M. A. Samad, M. D. Hossain and M. M. Alam "MHD free convection and mass transfer flow through a vertical oscillatory porous plate with Hall ion-slip currents and heat source in a rotating system", Procedia Engineering (Elsevier) 105, pp. 56 63, 2015

[10] Hayat, T. Ellahi, R., and Asghar, S. "Hall effect on unsteady flow due to non- coaxicially rotating disk and a fluid at infinity", chem., eng. Commun. 195 (80), 958-976, 2008.

[11] K. Manivannan, R. Muthucumarswamy, V. Thangaraj, "Radiation and chemical reaction effects on isothermal vertical oscillating plate with variable mass diffusion", Thermal science, vol.13, no.2, 2009.

[12] Rajput and Kanaujia, "MHD flow past a vertical plate with variable temperature and mass diffusion in the presence of Hall current", International Journal of Applied Science and Engineering, 14, 2, pp. 115-123, 2016.

[13] S.P. Dahake and A.V Dubewar "Effects of Radiation on Magnetohydrodynamic Convection Flow past an Impulsively Started Vertical Plate Submersed in a Porous Medium with Suction, International Journal of Computer Sciences and Engineering, vol. 2, Issues - 2, 2017.

The symbols involved in the above equations are mentioned in the appendix.

$$
\begin{aligned}
& A_{0}=\frac{u_{0}^{2} t}{v}, \quad A_{1}=\operatorname{erfc}\left[\frac{2 H i t-y \sqrt{P r}}{2 \sqrt{t}}\right], \quad A_{2}=\operatorname{erfc}\left[\frac{2 H i t+y \sqrt{P r}}{2 \sqrt{t}}\right], \\
& A_{3}=\left(-1-A_{17}+\exp (2 \sqrt{a} y)\left(A_{18}-1\right)\right), \\
& A_{4}=\left(1+A_{17}+\exp 2 \sqrt{a} y\left(A_{18}-1\right)\right), \\
& A_{5}=\left(-1+A_{19}+\exp (2 y) \sqrt{\frac{(a+H) \operatorname{Pr}}{\operatorname{Pr}-1}}\left(A_{20}-1\right)\right), \\
& A_{6}=\left(1+A_{21}+\exp (2 \sqrt{a} y)\left(1-A_{22}\right)\right), \\
& A_{15}=\exp \frac{a t}{S c-1}-y \sqrt{\frac{a S c}{S c-1}}, \quad A_{16}=\left(-1+\operatorname{erf}\left(\frac{y \sqrt{S c}}{2 \sqrt{t}}\right)\right), \\
& A_{17}=\operatorname{erf}\left(\sqrt{a t}-\frac{y}{2 \sqrt{t}}\right), \\
& A_{13}=\left(-1-A_{29}+\exp \left(2 y \sqrt{\left.\left.\frac{a S c}{S c-1}\right)\left(A_{30}-1\right)\right),}\right.\right. \\
& A_{12}=\left(1+A_{21}+\exp (2 y) \sqrt{\frac{a S c}{S c-1}}\left(A_{24}-1\right)\right), \\
& A_{10}=\left(1+A_{25}+\exp (2 H i y) \sqrt{\operatorname{Pr}\left(A_{25}\right.}\left(A_{26}-1\right)\right), \\
& A_{9}=\left(-1-A_{21}+\exp (2 H i y \sqrt{\operatorname{Pr}})\left(A_{26}-1\right)\right), \\
& \left.A_{27}+\exp \left(2 y \sqrt{\frac{(a+H) P r}{\operatorname{Pr} y}}\right)\left(A_{22}-1\right)\right),
\end{aligned}
$$




$$
\begin{aligned}
& A_{18}=\operatorname{erf}\left(\sqrt{a t}+\frac{y}{2 \sqrt{t}}\right), \quad A_{19}=\operatorname{erf}\left(\frac{y}{2 \sqrt{t}}-\sqrt{\frac{(a+H) t P r}{\operatorname{Pr}-1}}\right), \\
& A_{20}=\operatorname{erf}\left(\frac{y}{2 \sqrt{t}}+\sqrt{\frac{(a+H) t P r}{P r-1}}\right), \quad A_{21}=\operatorname{erf}\left(\frac{\sqrt{a t}-y}{2 \sqrt{t}}\right), \\
& A_{22}=\operatorname{erf}\left(\frac{\sqrt{a t}+y}{2 \sqrt{t}}\right), \quad A_{23}=\operatorname{erf}\left(\frac{y-2 t \sqrt{\frac{a S c}{S c-1}}}{2 \sqrt{t}}\right) \text {, } \\
& A_{24}=\operatorname{erf}\left(\frac{y+2 t \sqrt{\frac{a S c}{S c-1}}}{2 \sqrt{t}}\right), \quad A_{25}=\operatorname{erf}\left(H i \sqrt{t}-\frac{y \sqrt{P r}}{2 \sqrt{t}}\right), \\
& A_{26}=\operatorname{erf}\left(H i \sqrt{t}+\frac{y \sqrt{P r}}{2 \sqrt{t}}\right), \quad A_{27}=\operatorname{erf}\left(\sqrt{t} \sqrt{\frac{a+H}{\operatorname{Pr}-1}}-\frac{y \sqrt{\operatorname{Pr}}}{2 \sqrt{t}}\right), \\
& A_{28}=\operatorname{erf}\left(\sqrt{\frac{(a+H) t}{\operatorname{Pr}-1}}+\frac{y \sqrt{P r}}{2 \sqrt{t}}\right) \text {, } \\
& A_{29}=\operatorname{erf}\left(\frac{1}{2 \sqrt{t}}\left(\sqrt{\frac{a t}{S c-1}}-y \sqrt{S c}\right), \quad A_{30}=\operatorname{erf}\left(\frac{1}{2 \sqrt{t}}\left(\sqrt{\frac{a t}{S c-1}}+y \sqrt{S c}\right),\right.\right. \\
& A_{31}=\left(1+\operatorname{erf} \frac{2 \sqrt{a+b} t-y}{2 \sqrt{t}}\right), A_{32}=\left(1+\operatorname{erfc} \frac{2 \sqrt{a+b} t+y}{2 \sqrt{t}}\right) \text {, } \\
& A_{33}=1+A_{31}+\exp (2 \sqrt{a+b} y) A_{32}, \\
& B_{1}=\left(\frac{2 \sqrt{-H} t-\sqrt{\operatorname{Pr}} y}{2 \sqrt{t}}\right), B_{2}=\left(\frac{2 \sqrt{-H} t+\sqrt{\operatorname{Pr}} y}{2 \sqrt{t}}\right) \text {, } \\
& B_{3}=\sqrt{-H P r}, B_{4}=2 \sqrt{-H} t+\sqrt{\operatorname{Pr}} y \text {. } \\
& a=\frac{M}{1+m^{2}}(1-i m)+2 i \Omega \text {. }
\end{aligned}
$$

\section{AUTHORS PROFILE}

Dr. Uday Singh Rajput is a faculty member in the department of mathematics and astronomy, University of Lucknow, India. He has more than 25 years of teaching experience at UG and PG levels and also guided students for $\mathrm{Ph}$. D. degree. He has published more than 100 research articles. His research areas include MHD flows, Graph Theory and Operations Research.

Neetu Kanaujia is a research student in the department of mathematics and astronomy, University of Lucknow, India. 